\title{
Carcinoma de Células Escamosas de Labio Inferior: Asociación Entre Grado de Angiogénesis, Graduación Histológica y Frente de Invasión Tumoral
}

\author{
Lower Lip Squamous Cell Carcinoma: Relationship Between Angiogenesis Grade, Histological \\ Grading and Invasion Tumoral Front
}

"Mario Cantín López; "Iván Suazo Galdames; **Bernardo Venegas Rojas \& **Daniela Zavando Matamala

CANTÍN, L. M.; SUAZO, G. I.; VENEGAS, R. B. \& ZAVANDO, M. D. Carcinoma de células escamosas de labio inferior: Asociación entre grado de angiogénesis, graduación histológica y frente de invasión tumoral. Int. J. Morphol., 26(1):77-82, 2008.

RESUMEN: El carcinoma de células escamosas de la cavidad oral presenta una alta prevalencia en nuestro país, siendo el labio inferior el sitio más comúnmente afectado. El objetivo de este estudio fue determinar el grado de angiogénesis en el carcinoma de células escamosas de labio inferior, y su asociación con el grado de diferenciación según la Clasificación Internacional de Tumores y el Frente de Invasión Tumoral según el sistema de medición de Bryne, en pacientes con carcinoma de células escamosas de labio inferior, diagnosticados en los hospitales de Talca y Curicó, entre los años 1995 y 2005.

PALABRAS CLAVE: Carcinoma de células escamosas; Labio inferior; Angiogénesis; Graduación histológica; Frente de Invasión Tumoral.

\section{INTRODUCCIÓN}

El cáncer oral representa aproximadamente el 3 a $4 \%$ de todas las neoplasias malignas en el hombre (Sánchez et al., 2003; Martins et al., 2003). En la India y otros países asiáticos representan el $40 \%$ de los tumores malignos, mientras que en los países occidentales este índice varía de 3 a $5 \%$, estando entre los diez cánceres más comunes (Costa et al., 2002). En Chile, corresponde al 1,6\% del total de cánceres registrados. La mortalidad de cáncer oral y faríngeo, desde el año 1955 hasta 2002, representó un 1\%, con respecto a todos los cánceres, cuya tasa bruta fue en aumento de 0,9 a $1,3 \%$, por cada 100.000 habitantes. En relación a su morbilidad, en Chile representa aproximadamente el 1.6\% con respecto al total de cánceres (Riera \& Martínez, 2005).

El cáncer más común en la región oral es el carcinoma de células escamosas de la cavidad oral (CCECO), representando el $90 \%$ de las lesiones malignas (Gervasio et al., 2001), definiéndose como una neoplasia maligna de origen epitelial que se inicia a partir de células escamosas procedentes de los epitelios estratificados de la mucosa oral (Tumuluri et al., 2004). Esta lesión puede aparecer en diver- sas zonas de la cavidad oral, presenta una alta prevalencia en la región labial (Sugerman \& Savage, 2002; Luna-Ortiz et al., 2004; Khan et al., 2005), siendo el labio inferior el más comúnmente afectado (Kornevs et al., 2005; Al- Rawi \& Talabani, 2007; Conill et al., 2007; Vukadinovic et al., 2007).

Este carcinoma presenta una considerable variación histológica, aunque todos están compuestos por láminas o nidos de células con origen en el epitelio escamoso.

Broder (1920) clasificó los carcinomas basándose en los diferentes grados de diferenciación celular en: bien diferenciado, moderadamente diferenciado e indiferenciado (Kantola et al., 2001).

Las características histopatológicas de un tumor pueden diferir ampliamente de un área a otra y se considera que una de las informaciones diagnósticas más útiles pueden ser deducidas del Frente de Invasión Tumoral (FIT), que es donde residen presumiblemente las células tumorales más agresivas, determinando la naturaleza biológica del cáncer (Bryne, 1998).

\footnotetext{
* Unidad de Anatomía Normal, Facultad de Ciencias de la Salud, Universidad de Talca, Chile.

** Departamento de Estomatología, Facultad de Ciencias de la Salud, Universidad de Talca, Chile.
} 
Una vez que se forma la tumoración, se inicia la angiogénesis, proceso presente en células neoplásicas del tumor primario y se refiere a la formación de nuevos vasos sanguíneos producidos a partir del crecimiento y ramificación de vasos sanguíneos preexistentes (Nakaya et al., 2005).

Folkman (1974) demostró que los tumores sólidos no pueden crecer más allá de 2 a 3 milímetros de diámetro si no existe el aporte sanguíneo dado por la angiogénesis. Un aumento de la angiogénesis se puede visualizar en las zonas de respuestas reparativas o proliferativas tisulares (Suazo et al., 2007).

La angiogénesis participa en el desarrollo del tumor, invasión y metástasis (Lee et al., 1997; Okamoto et al., 2002) y es un factor asociado a la progresión y metástasis de tumores malignos. Este proceso es complejo y está mediado por factores reguladores. Estos factores inducen a la formación de nuevos vasos sanguíneos, los que se encargan del crecimiento del tumor (Naresh et al., 2001).

El propósito de este estudio es determinar el grado de angiogénesis en el carcinoma de células escamosas de labio inferior y su asociación con el grado de diferenciación según la Clasificación Internacional de Tumores y el Frente de Invasión Tumoral según el sistema de medición de Bryne, en pacientes con carcinoma de células escamosas de cavidad oral, diagnosticados en el Hospital Regional de Talca y Hospital Base de Curicó, Chile, entre los años 1995 y 2005.

\section{MATERIAL Y MÉTODO}

Se diseñó un estudio descriptivo, correlacional y retrospectivo. Las muestras fueron obtenidas de manera no probabilística por casos incidentes, desde los archivos del Hospital Regional de Talca y Hospital Base de Curicó, pertenecientes a pacientes diagnosticados entre los años 1995 y 2005.

Se utilizó un total de 17 muestras de carcinoma de células escamosas de la cavidad oral de labio inferior. En todos los casos, la histopatología era característica de los CCECO. La biopsia se obtuvo evitando zonas de necrosis o hemorragia. Las muestras obtenidas fueron cortadas y teñidas con Hematoxilina y Eosina (H \& E) para ser analizadas posteriormente.

El grado de diferenciación histológica del carcinoma de células escamosas de la cavidad oral, se obtuvo de acuerdo a la clasificación histológica según Broder modi- ficada por la OMS (Barnes et al., 2005). De acuerdo a su grado de diferenciación, se clasifican histológicamente en neoplasias bien diferenciadas (semejantes a sus células de origen), hasta una neoplasia mal diferenciada o anaplásica (que ha perdido los patrones celulares de su célula de origen), y siendo graduados como carcinomas bien diferenciados (G I), moderadamente diferenciados (G II) e indiferenciados (G III) (Oliveira et al., 2005).

El FIT del CCECO se obtuvo de acuerdo al sistema de graduación del FIT de Bryne, donde las muestras fueron clasificadas, otorgando puntuaciones individuales, de 1 a 4 puntos, a cada elemento morfológico en estudio, en base a las características individuales, grado de respuesta inmune, patrón de invasión tumoral, grado de queratinización y pleomorfismo nuclear. Este sistema de graduación de malignidad solamente evalúa las partes más invasivas del tumor. El score para cada parámetro es sumado y permite tener un score de malignidad total (Anneroth et al., 1987; Bryne et al., 1992; Bryne et al., 1995; Bryne).

Las muestras fueron incluidas en bloques de parafina, cortadas en secciones de $5 \mu \mathrm{m}$ y se les aplicó la tinción inmunohistoquímica estándar, para identificar el grado de angiogénesis, en base al anticuerpo monoclonal de ratón Collagen Type IV (NCL-COLL-IV), de especificidad para colágeno tipo IV humano, Clone PHM12 (Laboratorio Novocastra Ltda.).

Para determinar el grado de angiogénesis, las placas histológicas fueron observadas a través de un microscopio óptico marca Zeiss, modelo 0.25 Standard (Germany), con diferentes aumentos (32, 100, 200 y 400) siguiendo el procedimiento propuesto por Weidner et al. (1991), determinando la microdensidad vascular (MDV), identificando las áreas de mayor vascularización (hotspot) en el tejido tumoral (Tabla I). El conteo de vasos fue realizado con aumento de $400 \mathrm{X}\left(0,09 \mathrm{~mm}^{2}\right)$.

La información obtenida se analizó en base a tablas de contingencia, relacionando el grado de angiogénesis y los parámetros de Clasificación Histológica Internacional de Tumores y Sistema de Graduación del FIT de Bryne con todas sus variantes.

Para determinar el grado de asociación estadística entre las variables en estudio, se aplicó el test exacto de Fisher, considerando significativos valores iguales o menores a $0,05(\mathrm{p} \leq 0,05)$.

El procesamiento y cálculo de datos se realizó utilizando el programa STATA 9.0 para Windows. 


\section{RESULTADOS}

De un total de 17 muestras histológicas correspondientes a carcinoma de células escamosas de labio inferior, el $41,18 \%$ de los casos presentó un grado de angiogénesis leve, el mismo porcentaje un grado de angiogénesis moderada y el $17,66 \%$ de los casos un grado de angiogénesis avanzado (Tabla I).

Con respecto al grado de diferenciación celular (grado histológico), el 53\% de los casos se presentó como carcinoma bien diferenciado, el $41,2 \%$ presentó carcinoma del tipo moderadamente diferenciado. El 5,88\% de los casos restantes, correspondió a carcinoma del tipo indiferenciado (Tabla I).

En relación al FIT, el 29.4\% de los casos obtuvo un score total de 8, el 29,4\% el puntaje fue de 9, para el 23,5\% fue de 10; por último, sólo un 17,6\% obtuvo un score total de 11 puntos (Tabla I).

Tabla I. Características de las muestras de carcinoma de células escamosas de labio inferior obtenidas de pacientes de los Hospitales Regional de Talca y Base de Curicó, Chile. $\mathrm{n}=17$

\begin{tabular}{llc}
\hline Factor & $\mathbf{n}$ & $\%$ \\
Grado de angiogénesis & & \\
Leve & 7 & 41,18 \\
Moderado & 3 & 41,18 \\
Avanzado & & 17,66 \\
& & \\
Grado de diferenciación & 9 & 53,0 \\
Bien diferenciado & 7 & 41,2 \\
Moderadamente diferenciado & 1 & 5,88 \\
Indiferenciado & & \\
& & 29,4 \\
Frente de Invasión Tumoral & & 29,4 \\
Score 8 & 5 & 23,5 \\
Score 9 & 5 & 17,6 \\
Score 10 & 4 &
\end{tabular}

En la Tabla II se muestra la distribución de las muestra respecto del grado de angiogénesis y el frente de invasión tumoral, aplicándole el test exacto de Fisher para valores pequeños, se obtiene un valor de 0,594 , por lo que no existe evidencia para rechazar la hipótesis de igualdad entre el grado de angiogénesis y el score del FIT.
Tabla II. Nivel de asociación mediante test exacto de Fisher y el grado de angiogénesis y el Frente de Invasión Tumoral de Bryne, de las muestras de carcinoma de células escamosas de labio inferior obtenidas de pacientes de los Hospitales Regional de Talca y Base de Curicó, Chile. $\mathrm{n}=17$.

Frente de invasion tumoral

\begin{tabular}{cccccc}
\hline Angiogénesis & $\mathbf{8}$ & $\mathbf{9}$ & $\mathbf{1 0}$ & $\mathbf{1 1}$ & Total \\
\hline $\mathbf{1}$ & 2 & 3 & 2 & 0 & 7 \\
$\mathbf{2}$ & 2 & 1 & 1 & 3 & 7 \\
$\mathbf{3}$ & 1 & 1 & 1 & 0 & 3 \\
Total & 5 & 4 & 4 & 3 & 17 \\
& \multicolumn{5}{r}{}
\end{tabular}

En la Tabla III se presenta la distribución de las muestra respecto al grado de angiogénesis y al grado de diferenciación histológica. Aplicándole el test exacto de Fisher para valores pequeños, se obtiene un valor de 0,622 , lo que indica que no existe evidencia suficiente para rechazar la hipótesis de igualdad entre el grado de angiogénesis y el grado de diferenciación histológica.

Tabla III. Nivel de asociación mediante el test exacto de Fisher, entre grado de angiogénesis y diferenciación histológicade las muestras de carcinoma de células escamosas de labio inferior obtenidas de pacientes de los Hospitales Regional de Talca y Base de Curicó, Chile. $\mathrm{n}=17$.

\begin{tabular}{ccccc} 
& \multicolumn{4}{c}{ Grado de diferenciación } \\
Angiogénesis & $\mathbf{1}$ & $\mathbf{2}$ & $\mathbf{3}$ & Total \\
$\mathbf{1}$ & 5 & 2 & 0 & 7 \\
$\mathbf{2}$ & 3 & 3 & 1 & 7 \\
$\mathbf{3}$ & 1 & 2 & 0 & 3 \\
Total & 9 & 7 & 1 & 17 \\
\hline
\end{tabular}

Exacto Fisher $=0,622$ 
En nuestro estudio se esperaba encontrar una mayor densidad microvascular o grado de angiogénesis asociado al FIT de Bryne, a medida que aumentaba su score, al igual que en tumores de mayor indiferenciación o de menor grado de diferenciación histológica, categorizados de acuerdo a la Clasificación Internacional de Tumores. Sin embargo, de acuerdo al análisis de los datos obtenidos en el estudio, no encontramos una asociación estadísticamente significativa entre el grado de angiogénesis, FIT y el grado de diferenciación celular.

Al analizar la asociación entre el grado de angiogénesis y la Clasificación Histológica Internacional de Tumores, no se encontró una asociación estadísticamente significativa $(\mathrm{p}=0,622)$, contrariamente a lo visualizado por Oliveira et al. y Kurokawa et al. (2005), quienes observaron en carcinomas grados I y II, un menor grado de angiogénesis, con respecto a los carcinomas más indiferenciados.

Al analizar la asociación con el sistema FIT de Bryne, este resultó no tener una asociación significativa, contrariamente a lo esperado, ya que al aumentar el score del FIT debería haber una mayor proliferación de vasos sanguíneos, debido a que los elementos celulares y morfológicos necesitarían una mayor cantidad de sustentos nutricionales y fisiológicos. En 1992 Bryne, demostró que el FIT del carcinoma de piso de la cavidad oral es estadísticamente significativo en relación al grado de angiogénesis (Bryne et al., 1992.).

Estos resultados, pueden relacionarse, con la dificultad para distinguir entre vasos sanguíneos preexistentes y la formación de nuevos vasos sanguíneos, o la falta de diferenciación entre los vasos sanguíneos y linfáticos, observados microscópicamente (Shieh et al., 2004). El método más utilizado ha sido introducido por Weidner et al., que consiste en el conteo directo de los vasos sanguíneos, determinando un grado de angiogénesis y considerando este número como potencial angiogénico de la neoplasia (Ascani et al., 2005).

Al contrario de la gran mayoría de estudios revisados, en el presente no se logró demostrar una asociación entre el grado de angiogénesis y el grado de diferenciación y sistema de graduación del FIT, al ser analizado con la totalidad de los elementos morfológicos que lo constituyen. Resultados similares obtuvieron Tahan \& Stein (1995), concluyendo que el grado de angiogénesis en carcinoma de células escamosas de labio, no es un indicador de malignidad pronóstica.

Lo anterior permite sugerir la realización de estudio con una muestra mayor, considerando las tres variables en conjunto asociadas a parámetros clínicos.

CANTÍN, L. M.; SUAZO, G. I.; VENEGAS, R. B. \& ZAVANDO, M. D. Lower lip squamous cell carcinoma: Relationship between angiogenesis grade, histological grading and invasion tumoral front. Int. J. Morphol., 26(1):77-82, 2008.

SUMMARY: Squamous cell carcinoma of the oral cavity present a high prevalence in our country, become the lower lip the most common site affected. The aim of this study was determinated the angiogenesis grade in the lower lip squamous cell carcinoma and their relationship whit the histological grading according to the International Tumours Classification and the Invasion Tumoral Front according to the Bryne's system measuring in patients with lower lip squamous cell carcinoma diagnosed in the Talca's and Curico's Hospital between 1995 and 2005 years.

KEY WORDS: Squamous cell carcinoma; Lower lip, Angiogenesis; Histological grading; Invasion Tumoral Front.

\section{REFERENCIAS BIBLIOGRÁFICAS}

Al-Rawi, N. H. \& Talabani, N. G. Squamous cell carcinoma of the oral cavity: a case series analysis of clinical presentation and histological grading of 1,425 cases from Iraq. Clin. Oral Investig., 2007. [Epub ahead of print]

Anneroth, G.; Batsakis, J. \& Luna, M. Review of the literature and a recommended system of malignancy grading in oral squamous cell carcinoma. Scand. J. Dent. Res., 95(3):229-49, 1987.
Ascani, G.; Balercia, P.; Messi, M.; Lupi, L.; Goteri, G.; Filosa, A.; Stramazzotti, D.; Pieramici, T. \& Rubini, C. Angiogenesis in oral squamous cell carcinoma. Acta Otorhinolaryngol. Ital., 25(1):13-7, 2005.

Barnes, L.; Eveson, J. W.; Reichart, P. \& Sidransky, D. (Eds). World Health Organization Classification of Tumours. Pathology and Genetics of Head and Neck Tumours. Lyon (France), IARC Press, 2005. 
Broder, A. C. Squamous epithelium of the lip: a study of five hundred and thirty seven cases. JAMA, 74:65664, 1920 .

Bryne, M.; Jenssen, N. \& Boysen, M. Histological grading in the deep invasive front of T1 and T2 glottic squamous cell carcinomas has high prognostic value.Virchows Arch., 427(3):277-81, 1995.

Bryne, M.; Koppang, H. S.; Lilleng, R. \& Kjaerheim, A. Malignancy grading of the deep invasive margins of oral squamous cell carcinomas has high prognostic value. J. Pathol., 166(4):375-81, 1992.

Bryne, M. Is the invasive front of an oral carcinoma the most important area for prognostication? Oral Dis., 4(2):70-7, 1998.

Chidzonga, M. M. \& Mahomva, L. Squamous cell carcinoma of the oral cavity, maxillary antrum and lip in a Zimbabwean population: a descriptive epidemiological study. Oral Oncol., 42(2):184-9, 2006.

Conill, C.; Verger, E.; Marruecos, J.; Vargas, M. \& Biete, A. Low dose rate brachytherapy in lip carcinoma. Clin. Transl. Oncol., 9(4):251-4, 2007.

Costa, A. de L.; Pereira, J. C.; Nunes, A. A. \& Arruda, M. de L. Correlation between TNM classification, histological grading and anatomical location in oral squamous cell carcinoma. Pesqui. Odontol. Bras., 16(3):216-20, 2002.

Douglass, C. W. \& Gammon, M. D. Reassessing the epidemiology of lip cancer. Oral Surg. Oral Med. Oral Pathol., 57(6):631-42, 1984.

Folkman, J. Tumor angiogensis: role in regulation of tumor growth. Symp. Soc. Dev. Biol., 30(0):43-52, 1974.

Gervasio, O. L.; Dutra, R. A.; Tartaglia, S. M.; Vasconcellos, W. A.; Barbosa, A. A. \& Aguiar, M. C. Oral squamous cell carcinoma: a retrospective study of 740 cases in a Brazilian population. Braz. Dent. J., 12(1):57-61, 2001

Kantola, S.; Jokinen, K.; Hyrynkangas, K.; Mäntyselkä, P. \& Alho, O. P. Detection of tongue cancer in primary care. Br. J. Gen. Pract., 51(463):106-11, 2001.

Kayembe, M. K. \& Kalengayi, M. M. Histological and epidemiological profile of oral cancer in Congo (Zaire). Odontostomatol. Trop., 22(88):29-32, 1999.
Khan, M. I.; Hussain, M.; Jamil, M.; Arif, M.; Raza, A. \& Chaudhary, M. A. Squamous cell carcinoma lower lip an experience with Karapandzic technique. J. Coll. Physicians. Surg. Pak., 15(2):123-4, 2005.

Kornevs, E.; Skagers, A.; Tars, J.; Bigestans, A.; Lauskis, G.\& Libermanis, O. 5 year experience with lower lip cancer. Stomatologija, 7(3):95-8, 2005.

Kurokawa, H.; Zhang, M.; Matsumoto, S.; Yamashita, Y.; Tomoyose, T.; Tanaka, T.; Fukuyama, H. \& Takahashi, $\mathrm{T}$. The high prognostic value of the histologic grade at the deep invasive front of tongue squamous cell carcinoma. J. Oral Pathol. Med., 34(6):329-33, 2005.

Lee, A. H.; Happerfield, L. C.; Bobrow, L. G. \&, Millis, R. R. Angiogenesis and inflammation in invasive carcinoma of the breast. J. Clin. Pathol., 50(8):669-73, 1997.

Luna-Ortiz, K.; Guemes-Meza, A.; Villavicencio-Valencia, V. \& Mosqueda-Taylor, A. Lip cancer experience in Mexico. An 11-year retrospective study. Oral Oncol., 40(10):992-9, 2004.

Main, J. H. \& Pavone, M. Actinic cheilitis and carcinoma of the lip. J. Can. Dent. Assoc., 60(2):113-6, 1994.

Martins, G. B.; Reis, S. R. \& Silva, T. M. Collagen type I expression in squamous cell carcinoma of the oral cavity. Pesqui. Odontol. Bras., 17(1):82-8, 2003.

Nakaya, H.; Kawashiri, S.; Tanaka, A.; Noguchi, N.; Kato, K.; Hase, T. \& Yamamoto, E. Influences of angiogenesis and lymphangiogenesis on cancerous invasion in experimentally induced tongue carcinoma. J. Oral Pathol. Med., 34:87-92, 2005.

Naresh, K. N.; Nerurkar, A. Y. \& Borges A. M. Angiogenesis is redundant for tumour growth in lymph node metastases. Histopathology, 38(5):466-70, 2001.

Okamoto, M.; Nishimine, M.; Kishi, M.; Kirita, T.; Sugimura, M.; Nakamura, M. \& Konishi, N. Prediction of delayed neck metastasis in patients with stage I/II squamous cell carcinoma of the tongue. J. Oral Pathol. Med., 31(4):22733, 2002.

Oliveira, M. G.; Lauxen, I.; Martins, M.; Rados, P. V.; Jaeger, F.; Kaizer, M. R. \& Sant'Ana, M. Tongue squamous cell carcinoma: Relationship between argyrophilic nucleolar organizer regions(AgNORS) and histopathologic Grading. Appl. Cancer. Res., 25(1):20-4, 2005. 
Riera, S. P. \& Martínez, R. B. Morbilidad y mortalidad por cáncer oral y faríngeo en Chile. Rev. Méd Chile, 133(5):555-63, 2005.

Sánchez, J.; Gómez, D. \& Galera, H. Valor pronóstico del parámetro inmunohistoquímico p53 en los estadios I y II del carcinoma epidermoide de lengua móvil. Rev. Esp. Cirug. Oral y Maxilofac., 25(5):280-7, 2003.

Shieh, Y. S.; Lee, H. S.; Shiah, S. G.; Chu, Y. W.; Wu, C. W. \& Chang, L. C. Role of angiogenic and non-angiogenic mechanisms in oral squamous cell carcinoma: correlation with histologic differentiation and tumor progression. $J$. Oral Pathol. Med., 33(10):601-6, 2004.

Suazo, G. I. C.; Lara, S. M. C.; Cantín, L. M. G. \& Zavando, M. D. A. Efecto de la aplicación de láser de baja potencia sobre la mucosa oral lesionada. Int. J. Morphol.,25(3):523-8, 2007.

Sugerman, P. B. \& Savage, N. W. Oral cancer in Australia: 1983-1996. Aust. Dent. J., 47(1):45-56, 2002.

Tahan, S. R. \& Stein, A. L. Angiogenesis in invasive squamous cell carcinoma of the lip: tumor vascularity is not an indicator of metastatic risk. J. Cutan. Pathol., 22(3):236-40, 1995.

Tumuluri, V.; Thoma, G. A. \& Fraser, I. S. The relationship of proliferating cell density at the invasive tumour front with prognostic and risk factors in human oral squamous cell carcinoma. J. Oral Pathol. Med., 33(4):204-8, 2004.

Vukadinovic, M.; Jezdic, Z.; Petrovic, M.; Medenica, L. M. \& Lens, M. Surgical management of squamous cell carcinoma of the lip: analysis of a 10-year experience in 223 patients. J. Oral Maxillofac. Surg., 65(4):675-9, 2007.

Weidner, N.; Semple, J. P.; Welch, W. R. \& Folkman, J. Tumor angiogenesis and metastasis--correlation in invasive breast carcinoma. N. Engl. J. Med., 324(1):1-8, 1991.

Zitsch, R. P. Carcinoma of the lip. Otolaryngol. Clin. North. Am., 26(2):265-77, 1993.
Dirección para correspondencia:

Dr. Mario Cantín L.

Unidad de Anatomía Normal

Universidad de Talca

Avenida Lircay s/n Oficina N`104

Talca - CHILE

Fono $56-71-201682$

Email: mcantin@utalca.cl

Recibido : 29-08-2007

Aceptado: 26-11-2007 